\title{
Modeling the solid-to-plasma transition for laser imprinting in direct-drive inertial confinement fusion
}

\author{
G. Duchateau ${ }^{1}$, S.X. Hu ${ }^{2}$, A. Pineau ${ }^{1}$, A. $\mathrm{Kar}^{2}$, B. Chimier ${ }^{1}$, A. Casner ${ }^{1}$, \\ V. Tikhonchuk ${ }^{1}$, V. N. Goncharov ${ }^{2}$, P. B. Radha ${ }^{2}$, E. M. Campbell ${ }^{2}$ \\ ${ }^{1}$ Université de Bordeaux-CNRS-CEA, Centre Lasers Intenses et Applications, \\ UMR 510\%, 351 Cours de la Libération, 33405 Talence Cedex, France \\ 2 Laboratory for Laser Energetics, University of Rochester, 250 E. River Rd, Rochester, USA*
}

(Dated: April 10, 2019)

\begin{abstract}
Laser imprinting posses a potential danger for low-adiabat and high-convergence implosions in direct-drive inertial confinement fusion. Within certain direct-drive ICF schemes, a laser picket (prepulse) is used to conditioning the target to increase the interaction efficiency with the main pulse. Whereas initially the target is in a solid state (of ablators such as polystyrene) with specific electronic and optical properties, the current state-of-the-art hydro-codes assume an initial plasma state, which ignores the detailed plasma formation process. To overcome this strong assumption, a model describing the solid-to-plasma transition, eventually aiming at being implemented in hydro-codes, is developed. It describes the evolution of main physical quantities of interest, including the free electron density, the collision frequency, the absorbed laser energy, temperatures and pressure, during the first stage of the laser-matter interaction. The results show that a time about 100 picoseconds is required for the matter to undergo the phase transition, the initial solid state thus having an impact on the subsequent plasma dynamics. The nonlinear absorption processes (associated to the solid state) are also shown to have an influence on the thermodynamic quantities after the phase transition, leading to target deformations depending on the initial solid state. The negative consequences for the ICF schemes consist in shearing of the ablator and possibly non-negligible preliminary heating of the DT fuel.
\end{abstract}

PACS numbers:

\section{INTRODUCTION}

Inertial confinement fusion (ICF) has been actively pursued over the past four decades in laboratories [1$6]$. In the direct-drive ICF scheme [4-6], many intense laser beams are focused on a spherical capsule surface to ablate target material, thereby driving the deuteriumtritium contained capsule to implode through the socalled "rocket effect". The overlapped laser beams consisting of many speckles can give certain amount of intensity non-uniformity on the ICF target surface. Consequently, such spatial variations of laser intensity can cause nonuniform ablation on ICF target surface, leading to rough ablation surface and nonuniform shocks. This so-called laser-imprinting process [7] has been a concern since the beginning of direct-drive ICF, because the laserimprint induced ablation surface modulation can be exponentially amplified by Rayleigh-Taylor (RT) instability during the target acceleration phase. If not properly controlled, the laser-imprint induced RT instability can detrimentally degrade ICF target performance. Both experimental and simulation evidences [8-11] have indicated that laser imprint is the major cause for degrading implosion performance of low-adiabat $(\alpha=2-3.5)$ and high-convergence $\left(C_{R}>22\right)$ targets. Thus, understanding and mitigating laser-imprint effects are essential to

*Electronic address: guillaume.duchateau@u-bordeaux.fr the success of direct-drive ICF.

Understanding the laser-imprint effects on direct-drive ICF implosions relies on radiation-hydrodynamics simulations. However, the detailed process of laser-induced solid-to-plasma transition is currently missing in ICF hydro-codes. Instead, the current hydro-codes assume that the ICF target ablator is already in the plasma state at the very beginning $(t=0)$, i.e., having free-electron density $n_{e}$ larger than the critical electron density $\left(n_{c}\right)$ of the drive laser. For the commonly-used hydro-carbon polymer ablators such as polystyrene $(\mathrm{CH})$ and glow discharge polymer (GDP), this assumption of $n_{e}>n_{c}$ at $t=0$ is obviously invalid since these dielectric materials have no free (conduction) electrons at all before laser interaction. How laser speckles create free electrons to form plasmas should affect the laser imprinting process. For example, under the current wrong assumption of plasma state at $t=0$, the laser-induced shock pressure modulation $\delta P / P$ is linearly proportional to the laser intensity modulation $\delta I / I$. However, once the realistic multi-photon/tunneling ionization and impact ionization processes are correctly considered for the initial solidto-plasma transition, the target is expected to have a nonlinear response to the spatial laser-intensity modulation. This has been evidenced by recent two-dimensional VISAR measurements of spatial modulation in shock velocity, which showed a significant discrepancy between the current hydro-simulation and experiment [12]. Moreover, in contrast to an initial plasma state (currently used in hydrocodes), a dielectric material is initially transparent for the usual drive laser at a wavelength $\lambda=351 \mathrm{~nm}$. 
So laser energy could be deposited far from the target surface, with a spatial profile along the laser propagation direction. This is drastically different from the current treatment of assuming an initial plasma state in which the laser energy deposition is limited only to the overcritical target surface. Therefore, there is a need to develop a physics-based model to better capture the initial plasma formation process for direct-drive ICF simulations of laser imprinting.

This paper is devoted to develop such a model for better describing the initial laser-target interaction during the transition from the initial solid state to the plasma state (critical electron density formed), and the evolution of thermodynamic quantities as the electron and ionlattice (lattice of neutral atoms early in the interaction, going to a lattice of ions after ionization) temperature and pressure. It is first based on a microscopic description of the electron dynamics within a simplified band structure relevant to dielectric materials, allowing to describe the evolution of the produced free electron density with respect to time and space. Photo-ionization, impact ionization, and electron recombination are described. A Drude model then allows to evaluate the absorbed laser energy $[13,14]$. The involved electron collision frequency depends on the state of matter, accounting initially for only collisions with phonons, and finally collisions with ions in the plasma state. This absorbed laser energy is introduced as a source term in a twotemperature model to evaluate both electron and ionlattice temperatures. Finally, the previous description is coupled to a Beer-Lambert law to describe the laser intensity depletion inside the target. Numerical simulations have been performed with laser parameters relevant to the direct-drive ICF scheme. Despite some modeling approximations, this approach provides useful trends and conclusions within the present context. The results show clearly the influence of the initial solid state and laser intensity distribution on the macroscopic structure of the ablator and fuel.

In what follows we consider the interaction of a laser with a standard direct drive ICF target [5] containing a $8 \mu \mathrm{m}$ thick ablator, $50 \mu \mathrm{m}$ and $400 \mu \mathrm{m}$ thick solid and gas DT mixture, respectively. Namely, we estimate the spatial profile of absorbed energy along the laser propagation axis, in comparison with the current treatment in order to highlight the interest of modeling the solid-toplasma transition. We in particular focus on the amount of energy absorbed in the ablator bulk before entering the plasma state, and its consequences on the subsequent evolution. The so-called "shine-through" problem [15] is also addressed: at the early interaction where the laser intensity is not large enough, the ablator is transparent, possibly leading to an irradiation of the target core and preliminary undesirable heating. Eventually, our laserimprinting model will be implemented into radiationhydrodynamics codes for direct-drive ICF simulations.

The paper is organized as follows. The next Section II introduces the solid-to-plasma transition modeling for the laser energy absorption. All main ingredients described previously are detailed. Results and discussions are presented in Section III. The temporal evolutions of the free electron density and temperatures are first provided to exhibit the main mechanisms at play and demonstrate the reliability of the proposed model. Laser propagation across the ablator and energy deposition are then considered. By evaluating the induced pressure, it allows one to evaluate the mechanical deformations of the ablator and stresses at the ablator-fuel interface, exhibiting the consequences of the initial solid state on the generation of spatial nonuniformities. Consequences of considering an initial solid state (not plasma) on the DT fuel are finally provided. Conclusions and outlooks are drawn in Section IV.

\section{MODELING}

We consider here the early interaction of the laser picket with intensities about $10^{13}-10^{14} \mathrm{~W} / \mathrm{cm}^{2}$ with initially cold target on a timescale of 100 ps. In these conditions the hydrodynamic motion can be neglected and we focus out attention on the ionization and heating processes.

The first step of the laser interaction with a dielectric material is the absorption of photons by electrons, leading to the electron dynamics in the solid state of matter. The first stage consists of photo-ionization where valence electron are promoted to the bottom of the conduction band through multiphoton absorption or tunneling depending on both material and laser parameters. These promoted conduction electrons (or free electrons) can further absorb photons sequentially. So that they are promoted to higher energy states. When they reach an energy of the order of the band gap energy or more, their collisions with valence electrons can lead to a further ionization, the so-called impact ionization. Free electrons can also recombine to the valence band in the course of interaction. This whole dynamics can be described by multiple rate equations as [16]:

$$
\begin{array}{r}
\frac{\partial n_{0}}{\partial t}=\left(\frac{n_{v b 0}-n_{f e}}{n_{v b 0}}\right) W_{P I}+2\left(\frac{n_{v b 0}-n_{f e}}{n_{v b 0}}\right) \tilde{\alpha} n_{2} \\
-W_{1} n_{0}-n_{0} / \tau_{r} \\
\frac{\partial n_{1}}{\partial t}=W_{1} n_{0}-W_{1} n_{1}-n_{1} / \tau_{r} \\
\frac{\partial n_{2}}{\partial t}=W_{1} n_{1}-\left(\frac{n_{v b 0}-n_{f e}}{n_{v b 0}}\right) \tilde{\alpha} n_{2}-n_{2} / \tau_{r}
\end{array}
$$

where the electron population (density) $n_{i}$, with $i=$ $0,1,2$, corresponds to different energy states in the conduction band. $n_{0}$ corresponds to the bottom of the conduction band with an energy $E\left(n_{0}\right)=0$ by convention, while $E\left(n_{1}\right)=\hbar \omega$ and $E\left(n_{2}\right)=2 \hbar \omega$. The energy of two photons is sufficient for impact ionization with our parameters of polystyrene ablator interacting with UV laser of $\lambda=351 \mathrm{~nm}$, i.e. $E_{\max } \simeq 1.5 E_{g}$ [16] with a bandgap 
of $E_{g}=4.05 \mathrm{eV}$ and $\hbar \omega=3.54 \mathrm{eV}$. For this reason three electron populations are considered. $W_{P I}$ is the photoionization rate evaluated with the quantum Keldysh expression [17]. Such an approach is based on a temporal average over a few optical cycles which is consistent with the present slow variations of the laser pulse envelope. The Keldysh expression accounts for both multiphoton and tunneling ionization in the solid state depending on parameters. $\tilde{\alpha}$ is the rate for collisions between free and valence electrons leading to impact ionization. Its value is in the range of $\left[10^{14} s^{-1}, 10^{16} s^{-1}\right]$ for dielectric materials $[18-20]$. It is weighted by a factor 2 in Eq. (1) since the collision of an electron of state 2 with a valence electron leads to the production of two electrons in state 0 . To evaluate the order of magnitude of $\tilde{\alpha}$, it can be compared to the collision frequency used in the Drude model (widely used to evaluate optical properties of irradiated dielectrics by short laser pulses). The latter corresponds only to a scattering process where no significant change in the electronic structure takes place, and lies generally in the range of $10^{15} \mathrm{~s}^{-1}$. Since $\tilde{\alpha}$ corresponds to more important electronic rearrangement, its probability (cross section) should be significantly smaller. We thus choose $\tilde{\alpha}=10^{14} s^{-1}$. $W_{1}$ is the rate for one-photon absorption in the conduction band, which evolves linearly with the laser intensity. In general, electron-phonon-photon, electron-ion-photon, and direct interband [21] processes should contribute to its value. It should also depend on the energy of free electrons. Since a precise value of $W_{1}$ for $\mathrm{CH}$ is unknown, we use the silica parameters for illustration purpose (band gap of roughly $8 \mathrm{eV}$ ) assuming that the electron dynamics in the conduction band of dielectric materials is not very sensitive to the band gap value. In [16] for $\lambda=500 \mathrm{~nm}, W_{1}=3.5 \times 10^{-7} E_{L}^{2}$ in units of $s^{-1}$, where $E_{L}$ is the laser electric field. For electronphonon-photon collisional absorption, the rate scales as $\lambda^{4}$ [16] for moderate intensities. Thus, with $\lambda=351 \mathrm{~nm}$, we set $W_{1}=10^{-7} E_{L}^{2}$. Regarding the recombination, this model assumes that electrons can recombine within a timescale set to $\tau_{r}=1 \mathrm{ps}$ in accordance with standard values [20, 22, 23]. When going to a plasma state, the valence band is depopulated and the free electron density no longer evolves. To account for that, the coefficient $\left(n_{v b 0}-n_{f e}\right) / n_{v b 0}$ weights the ionization rates, where $n_{v b 0}=3 . \times 10^{22} \mathrm{~cm}^{-3}$ for $\mathrm{CH}$ is the initial electron density in the valence band, and $n_{f e}=n_{0}+n_{1}+n_{2}$ is the total free electron density. Note that we only consider single electron ionization, involving that the maximum free electron density is limited by the atomic density, which is close to the critical plasma density $\left(n_{c} \simeq 10^{22} \mathrm{~cm}^{-3}\right.$ for $\lambda=351 \mathrm{~nm}$ ). It is sufficient for our model which describes the transition of the ablator into the plasma state being opaque for the laser. It is worth mentioning that though several parameters and assumptions are used in this approach, they are consistent with the existing literature $[24,25]$ that has produced accurate results.

Going to the evaluation of the absorbed energy density, the collision frequency, $\nu_{c}$, accounting for collisions of
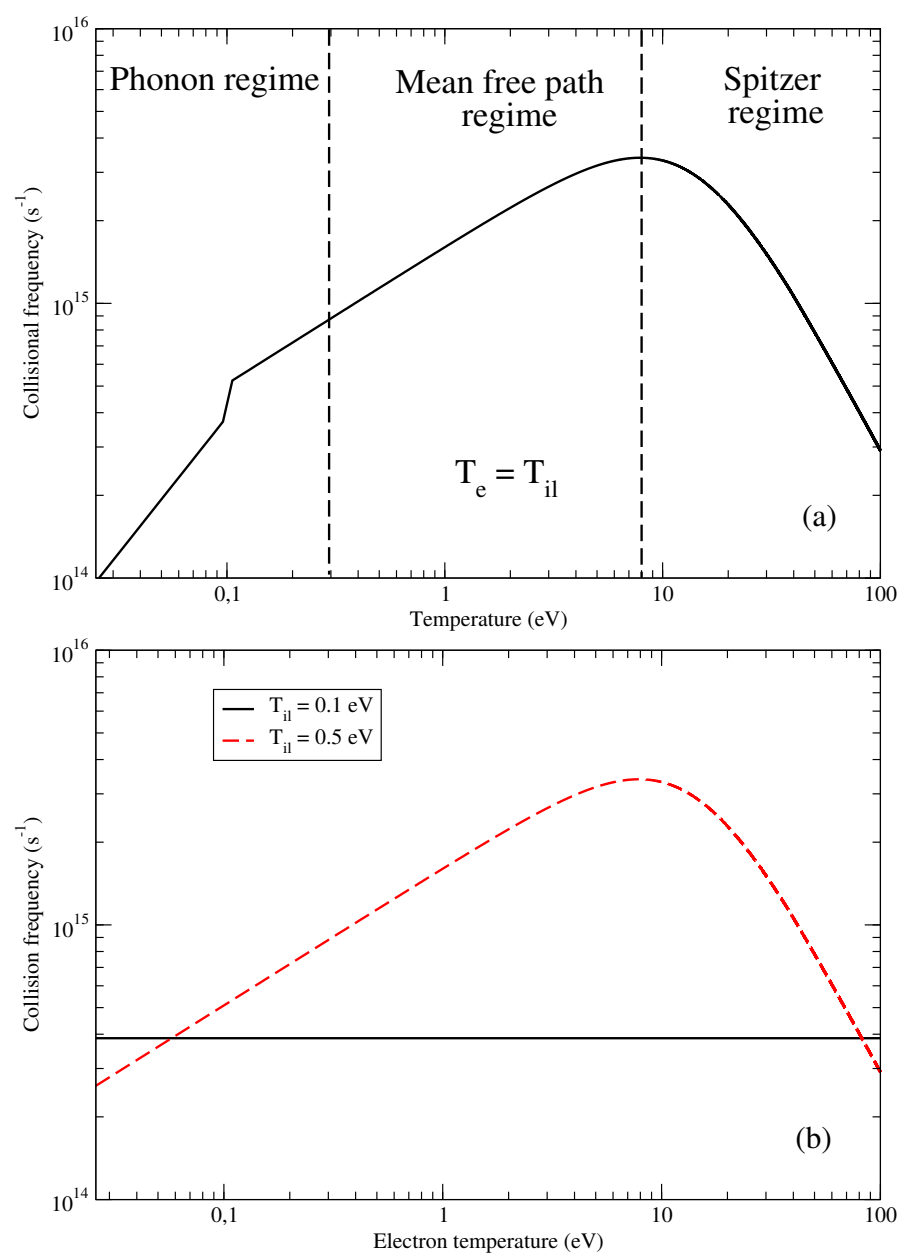

FIG. 1: Evolution of the collision frequency as a function of electron temperature in (a) the equilibrium case $\left(T_{e}=T_{i l}\right)$ and (b) for two constant ion-lattice temperatures of $0.1 \mathrm{eV}$ and $0.5 \mathrm{eV}$. In all cases $n_{f e}=10^{22} \mathrm{~cm}^{-3}$.

electrons with other particles has to be evaluated. It involves a momentum transfer rendering possible the photon absorption. Within the present approach, collisions with the ion-lattice are considered. In the limit cases, the solid state and the plasma state, collisions are driven by phonons and ions, respectively. The solid state is defined for not too high ion-lattice temperature, $T_{i l}$. Here we set $T_{i l}<0.1 \mathrm{eV}$ (corresponding to a fully melted state of $\mathrm{CH}$ polymer). For such temperatures, collisions are mainly due to phonons. The collision frequency then is assumed to evolve as $\nu_{p h}=\nu_{p h 0} T_{i l} / T_{0}[26,27] . \nu_{p h 0}$ is the electron-phonon collision frequency at room temperature $T_{0}=300 \mathrm{~K}$. It is set to $\nu_{p h 0}=10^{14} s^{-1}[21,26-28]$. The Spitzer expression for the elctron-ion collision frequency, $\nu_{p}$, is used for the full plasma state [29]. We use $Z \ln \Lambda=10$ for the illustration purpose, where for temperatures of tens of $\mathrm{eV}$ in the plasma state, both $Z$ and $\ln \Lambda$ are equal to a few unities. In the transition regime of electron and ion temperatures (typically in the few eV range close to the Fermi energy), the collision frequency 
is dominated by the mean free path of electrons with a probability of unity for momentum transfer at each collision [30]. This upper bound for the collision frequency reads $\nu_{m f p}=v_{e} n_{a}^{1 / 3}$ where $v_{e}$ is the electron thermal velocity and $n_{a}$ the atomic density, set to $3 . \times 10^{22} \mathrm{~cm}^{-3}$. For $T_{i l}>0.1 \mathrm{eV}$, the collision frequency is evaluated by the average expression $\nu_{a v}^{-1}=\nu_{m f p}^{-1}+\nu_{e-i}^{-1}$ where $\nu_{m f p}^{-1}$ ensures the transition between both asymptotic regimes.

The evolution of this collision frequency as a function of temperature, assuming the equilibrium $T_{e}=T_{i l}$ and $n_{f e}=10^{22} \mathrm{~cm}^{-3}$, is plotted in Fig. 1(a). Despite in the following results $T_{e}>T_{i l}$, this case is chosen for the illustration purpose allowing to exhibit all regimes at a glance. The vertical dashed lines separate the various regimes. Standard trends are retrieved [30] including the transition to the full plasma state for a temperature of the order of $10 \mathrm{eV}$. Note that the discontinuity in the collision frequency at $0.1 \mathrm{eV}$ is due to our stringent definition of the criterion to leave the solid state (phonons are removed since the material melts for a given temperature). Such a transition is obviously smoother but takes place in a rather narrow range of temperatures. Without changing the main conclusions of this work, we thus have chosen to keep this simple description. Figure 1(b) shows the evolution of the collision frequency with respect to the electron temperature for $T_{i l}=0.1 \mathrm{eV}$ and $T_{i l}=0.5 \mathrm{eV}$. In the first case, the collision frequency is constant since the matter is in the solid state. In the second case where the solid phase is removed, a similar behavior as the one of Fig. 1(a) is retrieved.

In order to determine the electron and ion-lattice temperatures, $T_{e}$ and $T_{i l}$, the two-temperature model is used [31]. By neglecting the transport on the present $100 \mathrm{ps}$ timescale for now (when the model is implemented into hydro-codes, the thermal conduction can be taken into account), this model reads:

$$
\begin{gathered}
C_{e} \frac{d T_{e}}{d t}=\frac{d U}{d t}-\frac{3}{2} k_{B} \frac{d n_{f e}}{d t} T_{e}-G\left(T_{e}-T_{i l}\right) \\
C_{i l} \frac{d T_{i}}{d t}=G\left(T_{e}-T_{i l}\right)
\end{gathered}
$$

Compared to the two-temperature model for metals, the additional term $\frac{3}{2} k_{B} \frac{d n_{f e}}{d t} T_{e}$ appears, accounting for the temporal evolution of the electronic heat capacity (due to the evolution of the free electron density). The heat capacities are $C_{e}=3 n_{f e} k_{B} / 2$ and $C_{i}=3 n_{a} k_{B} / 2$. These values pertain to temperatures of at least a few $\mathrm{eV}$ which are reached relatively early during the interaction. The electron-ion energy exchange factor $G$ is evaluated by $G=C_{e} \nu_{c} m_{e} / m_{i l}$. The electron to ion mass ratio weights the collision frequency to account for energy exchange $\left(\nu_{c}\right.$ accounts for momentum transfer). We set $m_{e} / m_{i l}=1 / 1836$ accounting for the fact that hydrogen atoms in $\mathrm{CH}$ mainly ensure the energy transfer. Doing so, we have checked that, after a fast $(<1$ ps) energy absorption in a solid state, the equilibrium is reached from a few to ten picoseconds which is a correct order of magnitude [20, 25]. The absorbed laser energy density per unit of time, $\partial_{t} U$, is described with the Joule heating term $\partial_{t} U=\vec{j} \cdot \vec{E}_{L}$ where $\vec{j}$ is the electron current density. By using the Ohm law, $\vec{j}=\sigma \vec{E}_{L}$ and the Drude model to evaluate the conductivity, $\sigma=\sigma_{0} /\left(1-i \omega \nu_{c}^{-1}\right)$ with $\sigma_{0}=e^{2} n_{f e} \nu_{c}^{-1} / m_{e}$, one obtains:

$$
\frac{\partial U}{\partial t}=\frac{e^{2} n_{f e} \nu_{c}}{m_{e}\left(\omega^{2}+\nu_{c}^{2}\right)} E^{2}
$$

To account for the laser propagation and predict the energy deposition along the direction of laser propagation, within the present conditions of relatively long time and spatial scales, a full Maxwell solver cannot be used due to its computational cost. Maxwell equations thus have to be simplified. First, we use the paraxial approximation consisting in neglecting second order derivatives. Since we consider the region of a laser speckle, the beam is mainly parallel in the interaction region and the propagation equation reduces to the Beer-Lambert law:

$$
v_{g} \frac{\partial I}{\partial z}=-\nu_{c} I-\left(\frac{n_{v b 0}-n_{f e}}{n_{v b 0}}\right) W_{P I} E_{g}
$$

with $v_{g}=c \sqrt{1-n_{f e} / n_{c}}, c$ being the speed of light in the matter. The first term on the right hand side accounts for the collisional absorption. The second one for the photoionization. By solving this equation for each time step, the temporal profile of the laser pulse and radial profile of the speckle are accounted for. It is worth noting that Eq. (7) remains valid as long as the electron density is lower than the critical plasma density. If not, the laser pulse can be reflected on the critical density, and Eq. (7) thus does not apply.

\section{RESULTS AND DISCUSSIONS}

\section{A. Temporal evolutions of physical quantities on the target surface}

The aim of this Section is to demonstrate the reliability of the model for the electron dynamics, the absorbed energy, and the temperatures evolution, by exhibiting values of these quantities which are reasonable. For that purpose, the laser propagation is not included, corresponding to consider the dynamics on the target surface.

To perform simulations with the model presented in section II, the used temporal intensity profile is shown in Fig. 2. It consists of a Gaussian shape with a duration of $100 \mathrm{ps}$ at full width at half maximum (FWHM). The maximum intensity is $10^{14} \mathrm{~W} / \mathrm{cm}^{2}$. Such an intensity profile is relevant of the pickets used in ICF experiments to conditioning the target [32]. For intensities in the $10^{13} \mathrm{~W} / \mathrm{cm}^{2}$ range, the photo-ionization rate is already large so that a full ionization is expected $[17,25]$. The 


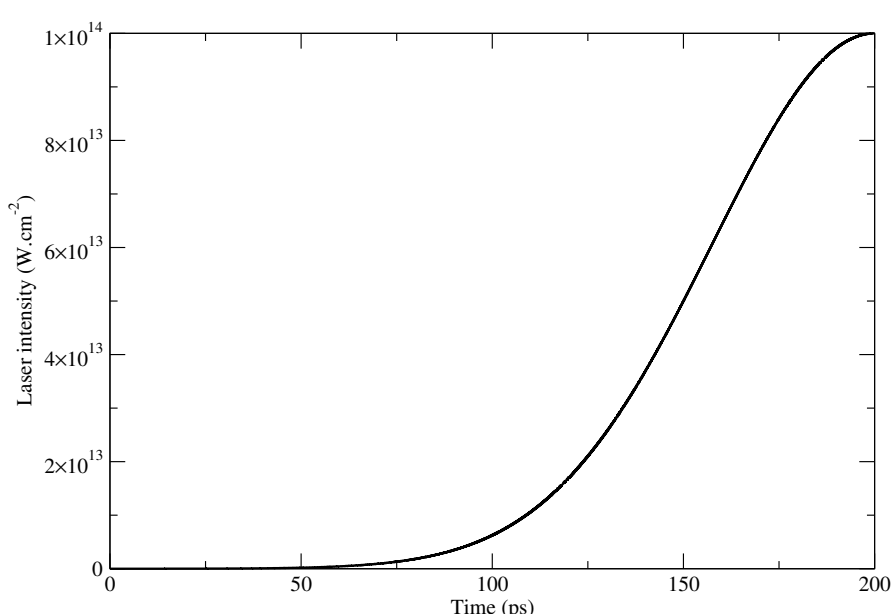

FIG. 2: Evolution of the laser intensity as a function of time. The second part of the pulse is not shown because simulations are stopped before.

simulations are thus stopped around $t \simeq 100 \mathrm{ps}$ where an overcritical plasma state $\left(n_{e}>n_{c}\right)$ is expected to be already formed.

Figure 3 shows the temporal evolution of (a) the free electron density, (b) both the electronic and ion-lattice temperatures, and (c) the collision frequency at the target front surface $(z=0)$ with all previously defined parameters. In all cases, the impact ionization process is taken into consideration or not in order to highlight its influence on the overall system dynamics. Regarding the electron density, without impact ionization, it evolves exponentially up to roughly $100 \mathrm{ps}$. This evolution follows the Gaussian shape of the laser pulse. After this time, the density saturates and reaches the maximum allowed value of $n_{a}$ within this model. When the impact ionization is switched on, a departure from the previous case is observed at 80 ps or so. At this time, the electron avalanche engages, leading the density to reach the maximum value in a shorter timescale, i.e. slightly before 100 ps. In particular the exponential behavior stands up to the saturation.

Due to the collisional absorption, following the electron density evolution, the electron temperature increases exponentially from $25 \mathrm{meV}(300 \mathrm{~K})$ to around $100 \mathrm{eV}$ on the $100 \mathrm{ps}$ timescale. In that case, temperatures are such that a plasma state is reached where the collision frequency decreases with respect to the electron temperature as shown in Fig. 3(c). As for the density, the electron temperature is lower when the impact ionization is switched off. Indeed, a smaller electron density leads to a smaller absorbed energy density. For such electron temperatures, electron-ion collisions may lead to multiple ionization, thus decreasing the temperature variations. Note that the slope has a discontinuity at $t \simeq 70$ ps. This is the signature of a change in the matter state where the electron-phonon collisions are replaced by the electron-ion collisions. This transition is well observed
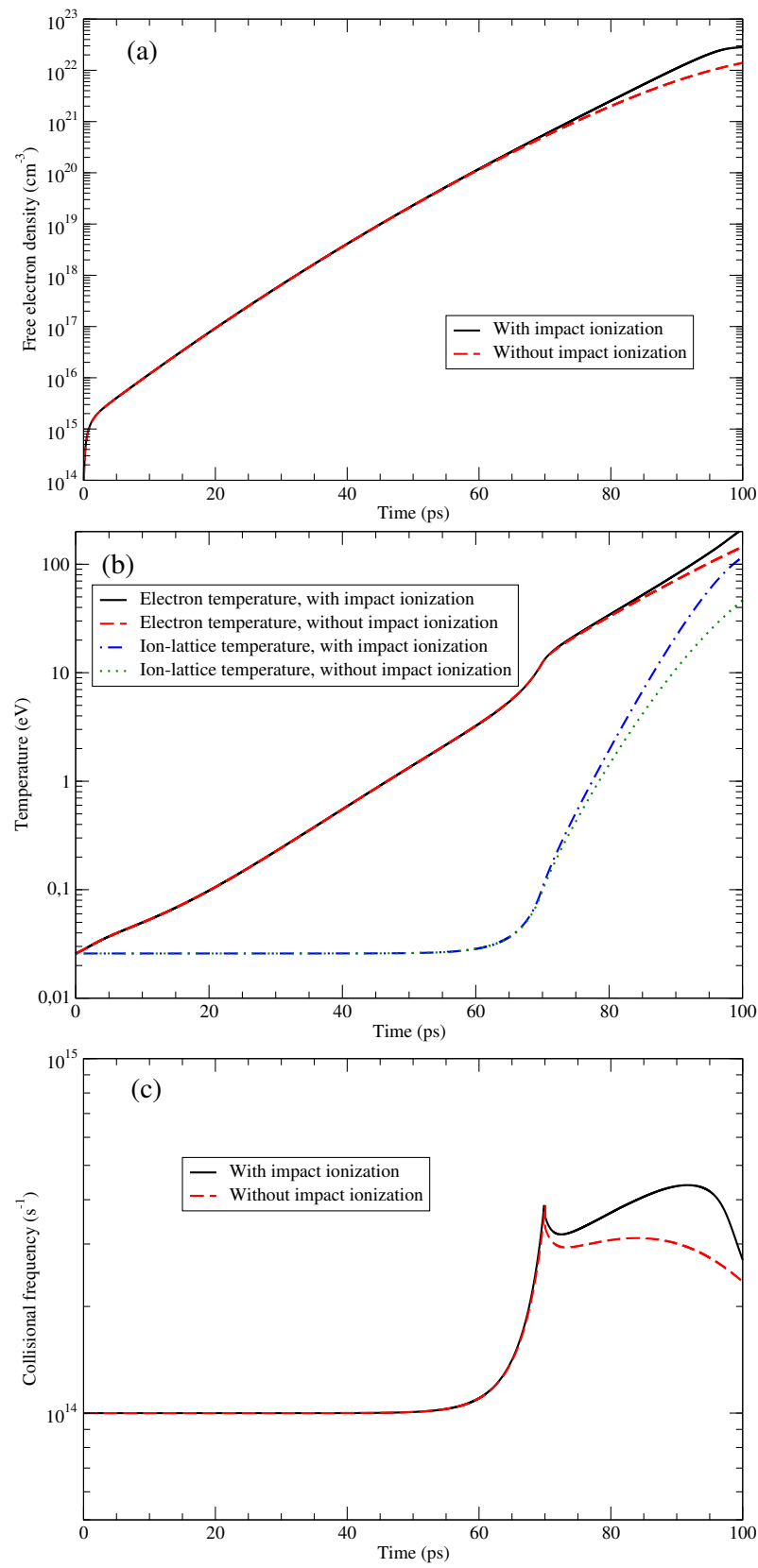

FIG. 3: Evolution of (a) the electron density, (b) the temperatures, and (c) the collision frequency as a function of time on the front surface $(z=0)$.

on the evolution of the collision frequency at $t \simeq 70 \mathrm{ps}$.

Regarding the ion-lattice temperature, as long as the electron temperature is not high enough, it remains close to the room temperature. It starts to increase when the electron temperature is of the order of $10 \mathrm{eV}$ at $t \simeq 65 \mathrm{ps}$. It then grows exponentially as the electron temperature rising. This two later regimes corresponds to a relatively low collision frequency $\left(10^{14} s^{-1}\right.$ for $\left.t<65 \mathrm{ps}\right)$, and a significantly larger one (for $t>70 \mathrm{ps}$ ), respectively. In the first regime, the electron-lattice energy transfer is not efficient while it has increased significantly in the 
second regime. As for the electrons, the impact ionization leads to higher ion-lattice temperatures but its influence is lost for the longest times. A break in the slope also appears at $t \simeq 70 \mathrm{ps}$ which indeed corresponds to $T_{i l}=$ $0.1 \mathrm{eV}$, i.e. the matter starts to undergo the solid-toplasma transition.

The evolution of the collision frequency as shown by Fig. 3(c) confirms the previous trends. As long as the solid state stands, it increases. Due to the first phase transition around $70 \mathrm{ps}$, the collision frequency evolution then is governed by the mean free path of electrons. It thus still continues to increase from a lower value (imposed by the state change) due to the electron temperature rise. Finally, it decreases when the plasma state is mainly reached where the Spitzer regime stands. Regarding the influence of the impact ionization, between $70 \mathrm{ps}$ and $100 \mathrm{ps}$, it leads to higher collision frequency since both electron density and temperatures are larger.

The following trends can be deduced from the previous study: (i) the electron temperature evolution is similar to the laser intensity envelope, (ii) The ion-lattice remains cold during a relatively long period of time, its temperature only increases strongly when the matter is entering the plasma state, (iii) photo-ionization dominates up to high densities of the order of $0.1 n_{c}$, only after the electron avalanche driven by the impact ionization engages. Such behaviors have already been reported in similar conditions [25] which makes the present modeling reliable. Note that the modeling parameters have been set to reasonable values also in accordance with the current state-of-the-art. Similar trends are expected by slightly varying the value of the modeling parameters. Also note that some of the used parameters may evolve with respect to the state of matter. Since there is no modeling to describe this evolution to our knowledge, they are set to the previous reasonable constant values for now.

Overall, the previous considerations have shed light on the dynamics of the ablator at the target front surface. The influence of the phase transition of the irradiated matter has been shown in particular. Theses evolutions in the target volume are now addressed in the following section.

\section{B. Laser propagation through the target bulk}

The objective of this section is to evaluate the bulk heating of the ablator before a full ionization takes place and plasma becomes opaque for the laser radiation.

The simulations performed in this section use the same model and parameters as previously but the laser propagation through the material is now included by further solving Eq. (7). To be consistent with the current direct drive ICF design [32], the thickness of $\mathrm{CH}$ shell is chosen to be $z_{\max }=8 \mu \mathrm{m}$. The evolutions of the laser intensity, the electron temperature, and the collision frequency as a function of both time and propagation distance $z$ are provided in Fig. 4. Regarding the laser intensity, at any instant, it decreases with respect to the propagated distance due to the absorption. However, the larger the laser intensity on the front surface (at a given time), the larger the intensity variations along the propagation axis. This behavior is due to the nonlinear evolution of the induced free electron density with respect to the intensity: the larger the intensity, the larger the ionization rate, and thus the stronger the laser absorption. That results in an almost constant intensity with respect to time at $z=8 \mu \mathrm{m}$, close to $1 \mathrm{TW} / \mathrm{cm}^{2}$ (for $t>70 \mathrm{ps}$ ). This value corresponds to the ionization threshold as it was discussed in the context of the so-called "shine-through" problem [15]. The two-photon absorption cross section is so small for intensities lower than this threshold that the produced electron density is not sufficient to affect the laser propagation (in terms of absorption due to ionization or heating of the free electrons). In addition, for such moderate intensities, the impact ionization rate is not large enough to engage an electron avalanche process [33].

The same trends are also observed for the electron temperature since it is roughly proportional to the electron density, the latter being a monotonic function of the laser intensity. Also the longer the propagation distance, the smaller the temperature; the longer the time (in the pulse), the stronger the temperature variations along the ablator thickness. When the critical density is reached $(t \simeq 90 \mathrm{ps})$, the temperature drops from $100 \mathrm{eV}$ on the front surface to a few tens of $\mathrm{eV}$ on the rear surface at $z=8 \mu \mathrm{m}$, whereas it is almost constant, of the order of a few $\mathrm{eV}$ to $10 \mathrm{eV}$, at $t \simeq 60-70 \mathrm{ps}$.

The latter behavior is in part due the collision frequency which remains the largest for $t \simeq 70-80 \mathrm{ps}$ along the whole propagation axis. For those times, both the temperature and the collision frequency have increased, but the ion-lattice temperature remains lower than the melting temperature. Considering the whole behavior of the collision frequency, similar trends with respect to time as presented in the previous section are retrieved whatever the traveled distance. However, above $z=2 \mu m$, after reaching the peak, it decreases slower with time compared to the behavior close to the front surface. This is due to the fact that temperatures are smaller. The absorption thus remains efficient, leading to a further decreasing channel for the intensity (in addition to the nonlinear absorption process). This overall explains why the variations in the various physical quantities are the smallest with respect to time for the longest traveled distances, and the smallest with respect to the distance for the shortest times: the present nonlinear system steers a smoother variation for the physical quantities for sufficiently long traveled distances or times.

To get more quantitative estimates and insights into the ICF conditions, cuts of the previous graphs are presented in Fig. 5. It shows the evolution of (a) the laser intensity, (b) the free electron density, (c) the electron and ion-lattice temperatures, and (d) the collision frequency as a function of the propagation distance at $t=90 \mathrm{ps}$ 

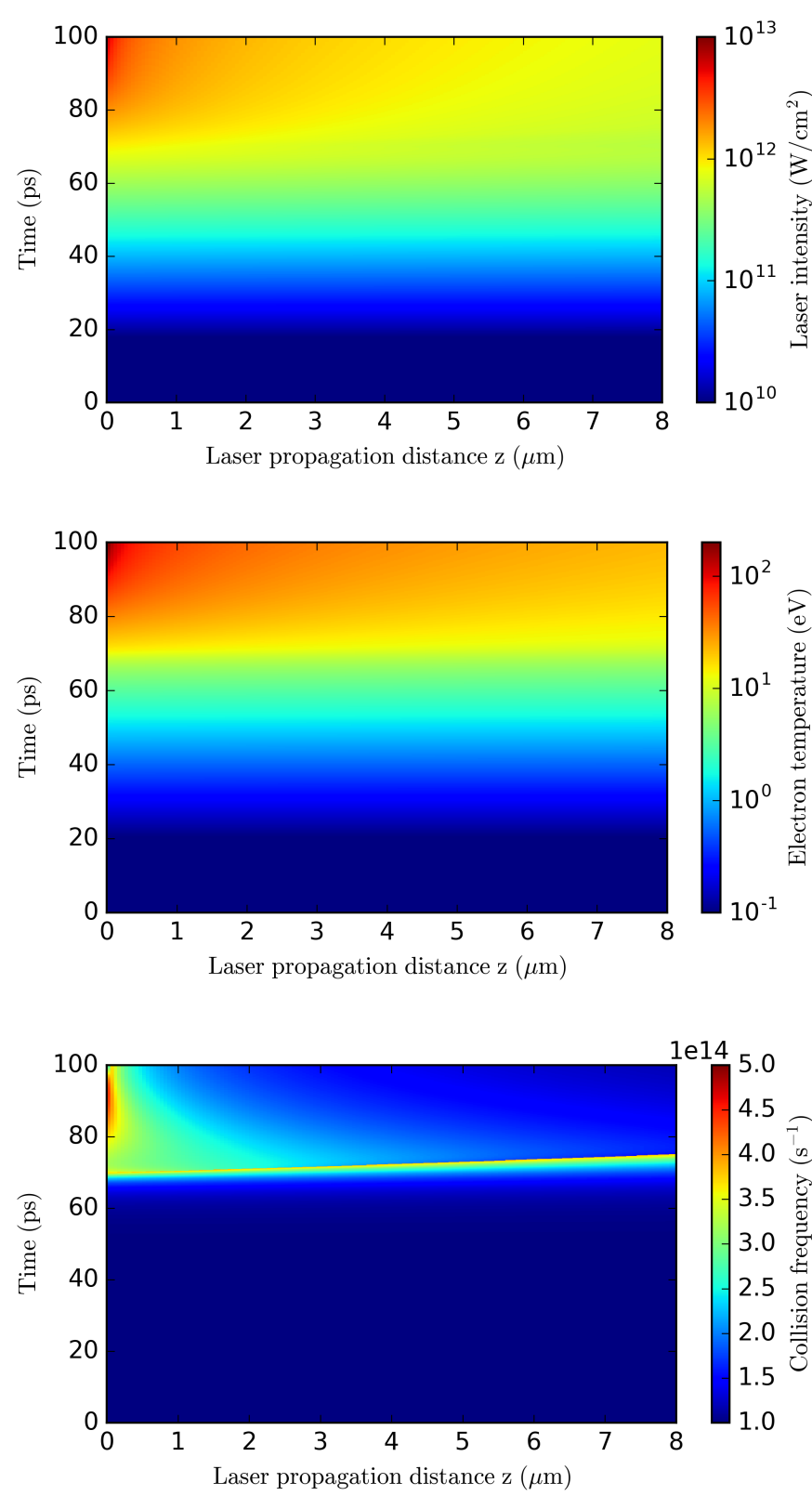

FIG. 4: Contour plots of laser intensity (top), electron temperature (middle), and collision frequency (bottom) as a function of time and the laser propagation axis $z$. It is noted that the laser starts at $z=0$ (target surface) and propagates along $z$ direction.

(when the critical density is reached on the front surface). All presented quantities decrease monotonically and regularly with respect to the propagation distance. In that case, the laser intensity on the front surface, close to $3 \mathrm{TW} / \mathrm{cm}^{2}$, is sufficiently small to ensure the same regime whatever the traveled distance: the electron density saturation is not reached and the induced temperatures are relatively small so that their variations are not too much important. In the course of propagation, the laser in-
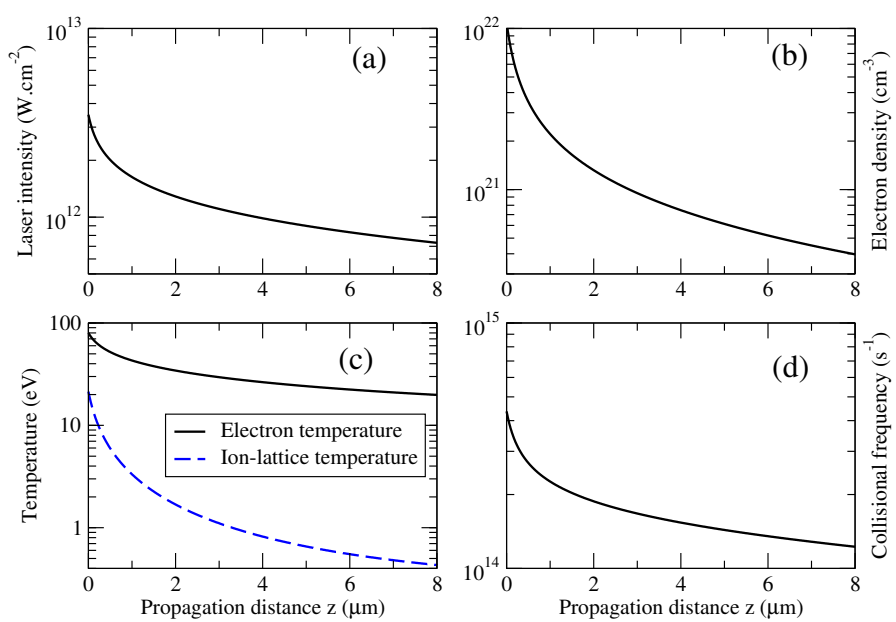

FIG. 5: At $t=90 \mathrm{ps,} \mathrm{evolution} \mathrm{of} \mathrm{(a)} \mathrm{the} \mathrm{laser} \mathrm{intensity,}$ (b) the free electron density, (c) the electron and ion-lattice temperatures, and (d) the collision frequency as a function of the propagation distance $\mathrm{z}$.

tensity decreases smoothly due to the absorption by the electrons. The free electron density thus also decreases due to lower intensities. The electron temperature thus follows the same trend. However its variations are less important since it is mainly driven by the collision frequency through the absorbed energy density $U$ (despite $U$ also includes the free electron density, its influence on the temperature is mainly canceled by the heat capacity which scales linearly with the electron density). Indeed the collision frequency only decreases by a factor of 4 because, in the Spitzer regime, its decrease imposed by the density is compensated by the decrease in temperature. Overall, the monotonic decrease in the collision frequency is mainly driven by the decrease in electron density.

\section{Consequences of the laser imprint on the $\mathbf{C H}$ ablator}

The laser imprint results from spatial laser inhomogeneities (speckles) which induce density and temperature gradients. The associated pressure thus leads to nonuniform deformations of both the $\mathrm{CH}$ shell and the $\mathrm{CH} / \mathrm{DT}$ interface. Such nonuniformities provide a seed to the development of RT instabilities. To evaluate the transverse deformations, the $1 \mathrm{D}$ description can be augmented to a $2 \mathrm{D}$ description through the following simple considerations. Since the ablator thickness is comparable to the speckle length (for a speckle width of $1 \mu \mathrm{m}$, the Rayleigh length is roughly $10 \mu m$ which is larger than the ablator thickness), a straightforward laser propagation can be considered, with negligible transverse losses. Then, knowing the intensity transverse profile at the entrance $z=0$, we can restitute the temperature and pressure profiles at the rear side $(z=8 \mu \mathrm{m})$ by solving Eq. (7) for various radial positions. Then an ideal gas equa- 


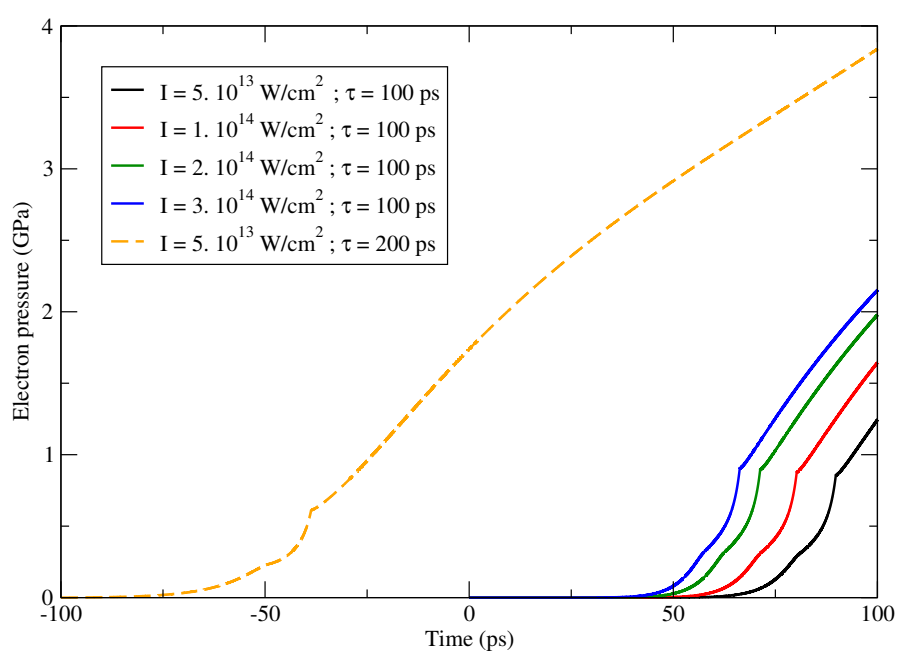

FIG. 6: Evolution of the electron pressure as a function of time at $z=8 \mu \mathrm{m}$ in the center of a speckle. Various laser parameters are used.

tion of state is used to get a first estimation of the electron pressure. Note that the ion-lattice pressure becomes equivalent when the temperature equilibrium is reached.

We first study the temporal evolution of the electronic pressure at $z=8 \mu m$ (the back surface of the $\mathrm{CH}$ shell) for various laser parameters. For the previous pulse duration, $\tau=100 \mathrm{ps}$, the electronic pressure temporal evolution at $z=8 \mu \mathrm{m}$ for various maximum intensities is provided by Fig. 6 (corresponding to the center of a speckle). The onset of the pressure growth takes place earlier for higher intensities. The pressure then increases almost linearly with respect to time. And as expected, the higher the laser intensity, the larger the pressure. However, by varying the intensity by a factor of 6 , the pressure only increases by $20 \%$. This small dependence on the intensity has been discussed previously. Within the present conditions, electronic pressure induced by a speckle are expected to be of a few GPa which is enough to induce significant deformations at the $\mathrm{CH} / \mathrm{DT}$ interface in a direct drive ICF target. Note that for the highest intensities and longest times, the pressures are slightly over-estimated since the critical density is reached.

To further exhibit how a laser speckle may imprint the target, a transverse direction to the laser propagation axis now is further considered. We assume a cylindrical symmetry, $r$ being the radial coordinate, where each physical processes at a given $r$ are independent of those of other radial position. This assumption is reliable if the transverse gradients of the electron density in particular are sufficiently small. The laser intensity profile thus should be relatively smooth which is the case with a Gaussian spatial profile of a speckle. The radial distribution of the various considered physical quantities are then obtained by performing the previous calculations, with some intensities relevant of the radial speckle distri- bution, independently from each other.

The following results have been obtained with a speckle size of $1 \mu \mathrm{m}$ FWHM, i.e. $\simeq 3 \lambda$, and a maximum intensity in the speckle center of $10^{14} \mathrm{~W} / \mathrm{cm}^{2}$. Figure 7 shows the evolution of the electron pressure with respect to both the propagation distance and the radial coordinate at $t=90 \mathrm{ps}$. Note that results for pressure higher than $100 \mathrm{GPa}$ are not shown to highlight the behaviors in the interface region around $z=8 \mu \mathrm{m}$. Fig 7 shows a high pressure region on the front surface which decreases along the radial direction as the laser intensity does. In the center of the speckle, the longer the propagation distance, the smaller the pressure. On the speckle edge, due to the threshold effect induced by nonlinear processes as discussed previously, the induced pressure is small (0.1 GPa) and is almost constant along the longitudinal coordinate. This results in strong pressure gradients on the front surface and relatively smooth on the interface. Such transverse pressure modulations may generate strong transverse acoustic waves with an amplitude depending on the longitudinal position $z$ (strong acoustic waves and no shock is expected in the transverse direction since the matter flow velocity $\sqrt{\Delta P / \rho} \simeq 1 \mathrm{~km} / \mathrm{s}$ is of the order of the sound speed). These acoustic waves may thus induce a local shearing of the plastic shell which may be detrimental for the subsequent implosion.

To more quantify these results on the interface, Fig. 8 shows the radial evolution of the pressure at $t=90 \mathrm{ps}$ within the same conditions as previously. The renormalized (to the maximum pressure at $r=0$ ) speckle intensity profile is also plotted as a reference. Despite the pressure decreases monotonically, two main regions can be observed. In the inner part $(r<0.5 \mu m)$, it is flatter than the intensity profile due to the above-mentioned smoothing influences. For longer radius, first a discontinuity in the slope can be observed which corresponds to conditions where the intensity is close to the previously discussed threshold of $1 \mathrm{TW} / \mathrm{cm}^{2}$. Crossing this intensity, the electron dynamics behavior becomes significantly different. The pressure then drops faster than the laser intensity with respect to the radial distance. It follows that $\delta P / P$ is a nonlinear function of $\delta I / I$, which is consistent with recent observations of the amplitude of shock velocity nonuniformity due to laser inhomogeneities [12].

In order to control these deformations, the laser parameters can be optimized. Here we consider a constant fluence. The case $I_{\max }=10^{14} \mathrm{~W} / \mathrm{cm}^{2}$ and $\tau=100 \mathrm{ps}$ is used as a reference. Calculations have been performed for $I_{\max }=5 \times 10^{13} \mathrm{~W} / \mathrm{cm}^{2}$ and $\tau=200 \mathrm{ps}$ providing the same fluence as the reference case. The resulting pressure is shown in Fig. 6. Compared to the reference case, obviously the onset takes place earlier (both maximum intensities are reached at $t=200 \mathrm{ps}$ ). The slope is smaller but since more time is allowed for heating the electron system, the final pressure is larger by a factor of $\sim 2$. It thus appears that, for a given fluence, shorter picket pulses are desirable. 


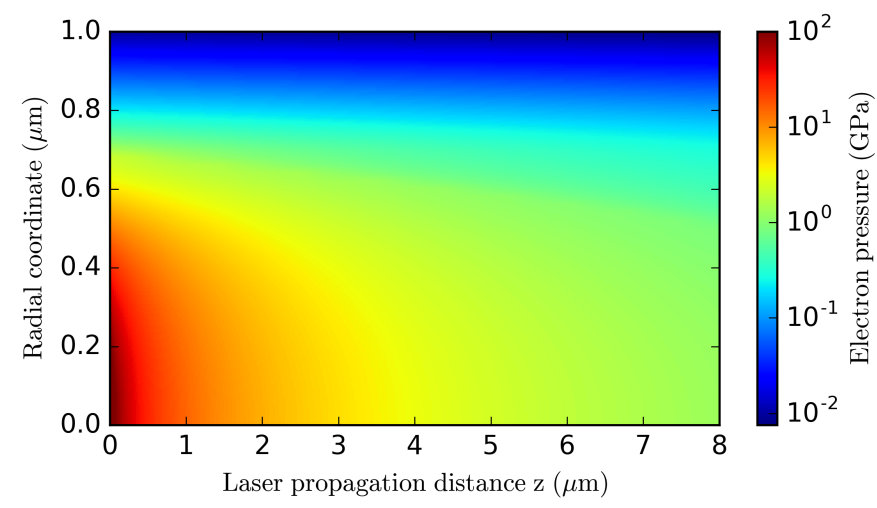

FIG. 7: Evolution of the electron pressure as a function of the laser propagation distance $z$ and the radial coordinate of a speckle, at $t=90 \mathrm{ps}$. The maximum intensity in space and time is $10^{14} \mathrm{~W} / \mathrm{cm}^{2}$.

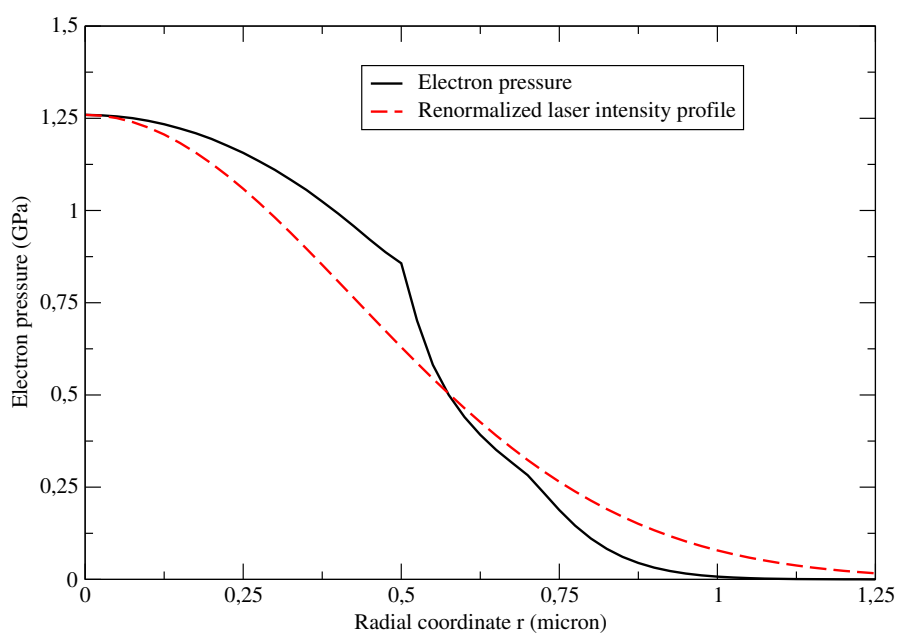

FIG. 8: At $t=90 \mathrm{ps}$ on the $\mathrm{CH} / \mathrm{DT}$ interface $(z=8 \mu \mathrm{m})$, evolution of the electron pressure as a function of the radial coordinate. The maximum incident intensity in time and space is $10^{14} \mathrm{~W} / \mathrm{cm}^{2}$.

The amount of energy of a laser picket which shines through the $\mathrm{CH}$ to the fuel then can be estimated from the present calculations. We have shown that the $\mathrm{CH}$ shell is mainly transparent for laser intensities lower than roughly $1 \mathrm{TW} / \mathrm{cm}^{2}$. Assuming the DT fuel consists of a shell of thickness of $\sim 50 \mu \mathrm{m}$ with a radius of $\sim 400 \mu \mathrm{m}$, an uniform irradiation with the threshold intensity during 100 ps corresponds to $\sim 0.7 \mathrm{~J}$ injected in the target. Due to the fact that several laser beams superpose inside the target, local intensities can be in excess of the threshold intensity, leading to a further absorption of the shinethrough irradiation by the inner part of the $\mathrm{CH}$ ablator. Assuming that only $10 \%$ of this shine-through energy is absorbed uniformly in the ablator, the associated energy density is $\sim 3 \mathrm{~kJ} / \mathrm{cm}^{3}$, leading to a temperature increase of the order of $\sim 2000 \mathrm{~K}$. Such an influence may lead the ablator to melt before the main laser pulse arrives, which could be detrimental for the ICF scheme as explained in more details in section IIID.

All the previous considerations have described the evolution of matter up to conditions where the critical plasma density is reached in order to ensure the reliability of the present modeling. This condition corresponds to the early formation of a plasma state at solid density or the so-called warm dense matter. Once this state is reached, with an electron density larger than the critical one, the laser reflection takes place in the vicinity of the front target surface. It is associated with a significant hydrodynamic expansion in the speckle irradiation region and a launch of longitudinal shock waves inside the ablator. Interaction of the shock with transverse acoustic waves then may shear the $\mathrm{CH} / \mathrm{DT}$ interface.

\section{Consequences on the DT fuel}

The previous estimations allow to draw conclusions regarding the integrity of the DT fuel following this early stage of the solid to plasma formation. The direct shinethrough irradiation should not have any influence on the solid DT since its bandgap is in excess of $10 \mathrm{eV}$. Indeed at least 3 photons are required to ionize the solid DT, the ionization rate is thus negligible with laser intensities of the order of $1 \mathrm{TW} / \mathrm{cm}^{2}$. The melting of the $\mathrm{CH}$ ablator at the $\mathrm{CH} / \mathrm{DT}$ interface, due to the shine-through irradiation, may be a first cause of loss of integrity of the DT fuel. Indeed due to heat conduction through the interface (the characteristic length of heat diffusion $2 \sqrt{D \Delta t}$ in solid DT within $1 \mathrm{~ns}$ is shorter than $1 \mathrm{~nm}$ ), the DT ice may start to undergo a phase transition at the interface before the main laser pulse arrives. That may results in a detachment of the $\mathrm{CH}$ shell from the ablator. Another detrimental effect could be due to the induced pressure by a laser speckle at the interface. First, the pressure gradient on the steep interface may deform it locally. Second, due to the spatial interface discontinuity, a shock wave (with moderate amplitude in the GPa range) is expected to be launched towards the DT fuel. The shock entropy of the order of $5 \mathrm{~kJ} / \mathrm{g}$ is ten times larger than the DT latent heat. It may lead to the fuel melting or vaporization. The laser interaction with the $\mathrm{CH}$ ablator may also induce a Bremsstrahlung irradition [34], leading to a further heating of the DT fuel through linear absorption. All these processes may lead the initial solid DT to expand at some places due to local phase transitions (inducing pressure gradients in the fuel). Generation of cracks could also be possible on the nanosecond timescale before the main laser pulse arrives since the shear strength of solid DT is only of the order of $1 \mathrm{MPa}$. These processes could be responsible for the loss of integrity of the solid DT fuel, thus modifying the target design expected to provide the best ICF conditions. 


\section{CONCLUSION AND OUTLOOKS}

In current state-of-the-art hydrocodes to simulate the inertial confinement fusion, a plasma state is assumed at the beginning of the interaction. All properties pertaining to the solid state of the ablator and fuel, as the insulating property, are not taken into consideration. For instance, the early absorption is modeled with inverse Bremsstrahlung whereas it is due to nonlinear processes as the multiphoton absorption resulting in electron transitions from the valence band to the conduction band of dielectric materials. Such current modeling may lead to over-estimate the early absorption and lead to spurious hydrodynamic behaviors or miss the energy deposition which take place deeper in the ablator, leading to incorrect predictions.

A model for the solid-to-plasma transition within the inertial confinement fusion framework has thus been developed for the first time. The main required ingredients have been used: electron dynamics in the solid state, absorption depending on the matter state (going from phonon assisted to ions), two-temperature model, and laser propagation. As a first modeling attempt within this context, the model is simplified by two assumptions: the hydrodynamic motion is neglected and the free electron density is limited by the critical plasma density. Despite its simplicity, this model allows us to draw first major conclusions: (i) roughly $100 \mathrm{ps}$ are required to induce a full plasma state in the speckle area of a laser picket, suggesting the current hydrocodes have to account for the solid-to-plasma transition to make reliable predictions; (ii) the spatial profiles of temperatures and pressures are influenced by early nonlinear absorption processes; (iii) detrimental effects of the initial solid state can be expected: local compression and shearing of the ablator and non negligible preliminary local heating of DT fuel, which may render inefficient the target design.

The present approach can be further extended by coupling it to a hydrodynamic code: it is not computationally time-consuming and could be easily adapted to model the laser propagation and energy deposition. Indeed the present approach of laser energy lost along a direction can be straightforwardly adapted to a curved trajectory as obtained within the standard geometrical optics, i.e. the ray tracing technique, or its complex version which allows to well know the laser intensity [35] which is mandatory to evaluate the rates of the nonlinear processes. The present model to describe the absorption during the solid-to-plasma transition is thus expected to be reliable on long timescale. Overall, a work dedicated to determine the conditions to switch between the present model and the standard inverse Bremsstrahlung absorption will have to be addressed.

Acknowledgments

GD thanks Patrick Mora for a fruitful discussion on the collisional absorption. Stéphane Coudert is also acknowledged for helping to make the contour plots with Python.
[1] S. W. Haan et al., Phys. Plasmas 18 , 051001 (2011).

[2] M. J. Edwards et al., Phys. Plasmas 18, 051003 (2011).

[3] R. Betti and O. A. Hurricane, Nat. Phys. 12, 435 (2016).

[4] D.D. Meyerhofer et al., Nuclear Fusion 51, 053010 (2011).

[5] V. N. Goncharov, T. C. Sangster, R. Betti, T. R. Boehly, M. J. Bonino, T. J. B. Collins, R. S. Craxton, J. A. Delettrez, D. H. Edgell, R. Epstein, R. K. Follet, C. J. Forrest, D. H. Froula, V. Yu. Glebov, D. R. Harding, R. J. Henchen, S. X. Hu, I. V. Igumenshchev, R. Janezic, J. H. Kelly, T. J. Kessler, T. Z. Kosc, S. J. Loucks, J. A. Marozas, F. J. Marshall, A. V. Maximov, R. L. McCrory, P. W. McKenty, D. D. Meyerhofer, D. T. Michel, J. F. Myatt, R. Nora, P. B. Radha, S. P. Regan, W. Seka, W. T. Shmayda, R. W. Short, A. Shvydky, S. Skupsky, C. Stoeckl, B. Yaakobi, J. A. Frenje, M. Gatu-Johnson, R. D. Petrasso, and D. T. Casey, Phys. Plasmas 21, 056315 (2014).

[6] R. S. Craxton, K. S. Anderson, T. R. Boehly, V. N. Goncharov, D. R. Harding, J. P. Knauer, R. L. McCrory, P. W. McKenty, D. D. Meyerhofer, J. F. Myatt, A. J. Schmitt, J. D. Sethian, R. W. Short, S. Skupsky, W. Theobald, W. L. Kruer, K. Tanaka, R. Betti, T. J. B. Collins, J. A. Delettrez, S. X. Hu, J. A. Marozas, A. V. Maximov, D. T. Michel, P. B. Radha, S. P. Regan, T. C. Sangster, W. Seka, A. A. Solodov, J. M. Soures,
C. Stoeckl, and J. D. Zuegel, Phys. Plasmas 22, 110501 (2015)

[7] R. Ishizaki and K. Nishihara, Phys. Rev. Lett. 78, 1920 (1997); Phys. Rev. E 58, 3744 (1998).

[8] S. X. Hu, P. B. Radha, J. A. Marozas, R. Betti, T. J. B. Collins, R. S. Craxton, J. A. Delettrez, D. H. Edgell, R. Epstein, V. N. Goncharov, I. V. Igumenshchev, F. J. Marshall, R. L. McCrory, D. D. Meyerhofer, S. P. Regan, T. C. Sangster, S. Skupsky, V. A. Smalyuk, Y. Elbaz, and D. Shvarts, Phys. Plasmas 16, 112706 (2009); S. X. Hu, V. N. Goncharov, P. B. Radha, J. A. Marozas, S. Skupsky, T. R. Boehly, T. C. Sangster, D. D. Meyerhofer, and R. L. McCrory, Phys. Plasmas 17, 102706 (2010).

[9] P. B. Radha, C. Stoeckl, V. N. Goncharov, J. A. Delettrez, D. H. Edgell, J. A. Frenje, I. V. Igumenshchev, J. P. Knauer, J. A. Marozas, R. L. McCrory, D. D. Meyerhofer, R. D. Petrasso, S. P. Regan, T. C. Sangster, W. Seka, and S. Skupsky, Phys. Plasmas 18, 012705 (2011).

[10] S. X. Hu, D. T. Michel, A. K. Davis, R. Betti, P. B. Radha, E. M. Campbell, D. H. Froula, and C. Stoeckl, Phys. Plasmas 23, 102701 (2016).

[11] D. T. Michel, S. X. Hu, A. K. Davis, V. Yu. Glebov, V. N. Goncharov, I. V. Igumenshchev, P. B. Radha, C. Stoeckl, and D. H. Froula, Phys. Rev. E 95, 051202(R) (2017).

[12] J. Peebles, S.X. Hu, V. N. Goncharov et al., Phys. Rev. 
Lett. (submitted).

[13] E. G. Gamaly, A. V. Rode, B. Luther-Davies, and V. T. Tikhonchuk, Phys. Plasmas 9, 949 (2002)

[14] G M Petrov and J Davis, J. Phys. B: At. Mol. Opt. Phys. 41, 025601 (2008)

[15] D. H. Edgell, W. Seka, R. E. Bahr, T. R. Boehly, and M. J. Bonino, Phys. Plasmas 15, 092704 (2008)

[16] B. Rethfeld B, Phys. Rev. Lett. 92, 187401 (2004)

[17] L. V. Keldysh, Sov. Phys.-JETP 20, 1307 (1965)

[18] J. R. Penano, P. Sprangle, B. Hafizi, W. Manheimer, and A. Zigler, Phys. Rev. E 72, 036412 (2005)

[19] B. C. Stuart, M. D. Feit, S. Herman, A. M. Rubenchik, B. W. Shore, and M. D. Perry, Phys. Rev. B bf 53, 1749 (1996)

[20] R. R. Gattass and E. Mazur, Nature Photonics 2, 219 (2008)

[21] L. Barilleau, G. Duchateau, B. Chimier, G. Geoffroy and V. Tikhonchuk, J. Phys. D: Appl. Phys. 49, 485103 (2016)

[22] G. Duchateau, M. D. Feit, and S. G. Demos, J. Appl. Phys. 111, 093106 (2012)

[23] G. Duchateau, M. D. Feit, and S. G. Demos, J. Appl. Phys. 115, 103506 (2014)

[24] B. Rethfeld et al., J. Phys. D: Appl. Phys. 50, 193001
(2017)

[25] N. M. Bulgakova, V. P. Zhukov, Y. P. Meshcheryakov, L. Gemini, J. Brajer, D. Rostohar, and T. Mocek, J. Opt. Soc. Am. B 31, C8-C14 (2014)

[26] E. G. Gamaly, Physics Reports 508, 91 (2011)

[27] E. G. Gamaly and A. V. Rode, J. Opt. Soc. Am. B 31, C36 (2014)

[28] A. Kaiser, B. Rethfeld, M. Vicanek, and G. Simon G, Phys. Rev. B 61, 11437 (2000)

[29] L. Spitzer, Jr., Physics of Fully Ionized Gases, Interscience Publishers, New York, 1956

[30] K. Eidmann, J. Meyer-ter-Vehn, and T. Schlegel, S. Hller, Phys. Rev. E 62, 1202 (2000)

[31] S.I. Anisimov, B. Kapeliovich, T. Perelman, Sov. Phys. JETP 39, 375 (1974)

[32] V. N. Goncharov et al., Phys. Rev. Lett. 104, 165001 (2010).

[33] B. Rethfeld, Phys. Rev. B 73, 035101 (2006)

[34] G. M. Petrov, J. P. Palastro, and J. Peano, Phys. Rev. E 95, 053209 (2017)

[35] A. Colaitis, G. Duchateau, P. Nicolai, and V. Tikhonchuk, Phys. Rev. E 89, 033101 (2014) 